\title{
DIC DEFORMATION ANALYSES OF MG SPECIMENS AT ELEVATED TEMPERATURES
}

\author{
Thomas Lehmann ${ }^{1}$, Julia Müller ${ }^{1}$ Jörn Ihlemann ${ }^{1}$ \\ 1 Chemnitz University of Technology, Professorship of Solid Mechanics, Reichenhainer Straße 70, \\ 09126 Chemnitz, Germany. E-mail: thomas.lehmann@mb.tu-chemnitz.de
}

\section{Introduction}

Mg alloys have become more and more important for application in lightweight structures. During manufacturing processes by forming of textured AZ31 semi-finished products the difference of the yield strengths in tension and compression has to be considered [1]. This effect is caused by twinning mechanisms (tension twin system, compression twin system) in textures and the activation of additional sliding planes at elevated temperatures [2]. To investigate the influence of the yield strength differences in tension and compression during bending deformation, experimental and numerical investigations were performed. In this contribution the experimental analysis of the material behavior of AZ31 in bending tests at elevated temperatures is demonstrated. The focus is on experimental deformation analyses and testing of different evaluation methods of the displacement fields.

\section{Experimental setup and procedure}

For the bending tests twin roll casted AZ31 (TRC) sheet material of two different thicknesses $t=2.5$ and $1.5 \mathrm{~mm}$ was used. The specimens were separated from the semi-finished products by wire eroding. The geometry, testing temperatures and number of analyzed specimens are given in Tab. 1 .

\begin{tabular}{|c|c|c|c|}
\hline $\begin{array}{c}\text { Semi- } \\
\text { finished } \\
\text { product }\end{array}$ & $\begin{array}{c}\text { Specimen } \\
\text { geometry } \\
\boldsymbol{w} \times \mathbf{t} \text { x } \boldsymbol{l} \text { [mm] }\end{array}$ & $\begin{array}{c}\text { Testing } \\
\text { tempera- } \\
\text { ture }\left[{ }^{\circ} \mathbf{C}\right]\end{array}$ & $\begin{array}{c}\text { Number } \\
\text { of } \\
\text { specimens }\end{array}$ \\
\hline AZ31 & $5 \times 2.5 \times 15$ & 250 & 2 \\
\cline { 2 - 4 } (TRC) & & 350 & 3 \\
\cline { 2 - 4 } sheets & $5 \times 1.5 \times 15$ & 250 & 3 \\
\cline { 3 - 4 } & & 350 & 3 \\
\hline
\end{tabular}

Tab. 1. Subset of testing parameters.

The load was applied by a punch while the specimen was clamped, see Fig. 1. Bending specimen and device were surrounded by a temperature chamber and assembled in a $100 \mathrm{kN}$
Zwick/Roell testing machine. Because of low loads, forces were measured using a $5 \mathrm{kN}$ GTM load cell.

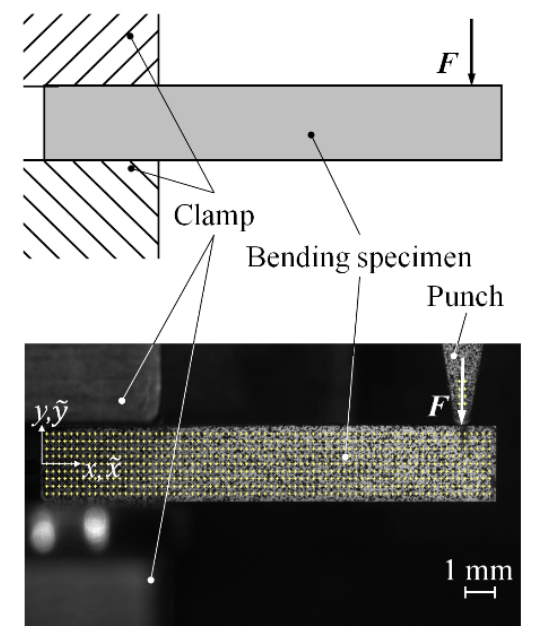

Fig. 1. Bending test, above: load case, below: specimen and device, virtual measuring pattern at the surface.

The specimens were loaded with punch displacements $4 \mathrm{~mm}$ (for $t=2.5 \mathrm{~mm}$ ) and $2.8 \mathrm{~mm}$ (for $t=1.5 \mathrm{~mm}$ ) and a velocity of $2.5 \mathrm{~mm} / \mathrm{s}$. The deformation analyses were performed using 2D digital image correlation (DIC) with the software VEDDAC (CWM GmbH, Chemnitz). Hence, a CCD monochrome camera, connected to the measuring laptop, with telecentric lens was applied. A black and white speckle pattern at the specimens surface improved the DIC algorithm. For image sequence acquisition a frame rate of $10 \mathrm{~Hz}$ was used. During the DIC analyses subsequent to the bending tests a virtual measuring pattern was defined at the surface of the specimens, see Fig. 1 (below).

\section{Evaluation method}

The DIC displacement data was extended using linear extrapolation to the edges and interpolation between the measuring points. Due to noise of the experimental data the displacement values were smoothed. Additionally, for the calculation of strain tensors differentiability of the determined 


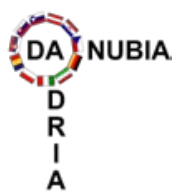

34th Danubia-Adria Symposium on Advances in Experimental Mechanics

University of Trieste, Italy, 2017 displacements was necessary. Therefore, the 2D displacement data at the specimen surface was approximated by two different methods using the software Matlab. By means of the approximation, functions of the displacement vector field $\mathbf{u}(\tilde{x}, \tilde{y})=\left[u_{x}(\tilde{x}, \tilde{y}) u_{y}(\tilde{x}, \tilde{y})\right]^{\mathrm{T}}$ in dependency of the Lagrange coordinates were obtained. The first variant was approximation by global polynomial surfaces [3]. Variations of the polynomial degree $p$ showed that $p=8$ for $u_{x}$ and $u_{y}$ is suitable to represent the specific displacement fields. The second variant used was approximation by cubic B-spline surfaces. The deformation gradient $\mathbf{F}$ is determined by the identity tensor $\mathbf{I}$ and the displacement vector $\mathbf{u}$ using the following equation:

$$
\mathbf{F}=\mathbf{I}+(\tilde{\nabla} \otimes \mathbf{u})^{\mathrm{T}} .
$$

Based on the deformation gradient the Lagrangian Hencky tensor $\mathbf{H}$ is given by

$$
\mathbf{H}=0.5 \ln \left(\mathbf{F}^{\mathrm{T}} \mathbf{F}\right) \text {. }
$$

\section{Results}

The evaluation of the displacement data by global polynomial approximation is demonstrated by an example of a deformed specimen with thickness $t=2.5 \mathrm{~mm}$ at testing temperature $350^{\circ} \mathrm{C}$ and a punch displacement of $4 \mathrm{~mm}$. In Fig. 2 the $x$ coordinate $H_{x x}$ of the Lagrangian Hencky tensor is plotted versus the Euler coordinates $x$ and $y$. In the deformation zone the method provides a good demonstration of the strain distribution. At the left edge significant strain values appear, which are unrealistic because of nearly deformation free conditions inside the clamp. This effect occurs due to "overshooting" of the polynomial functions in edge sections.

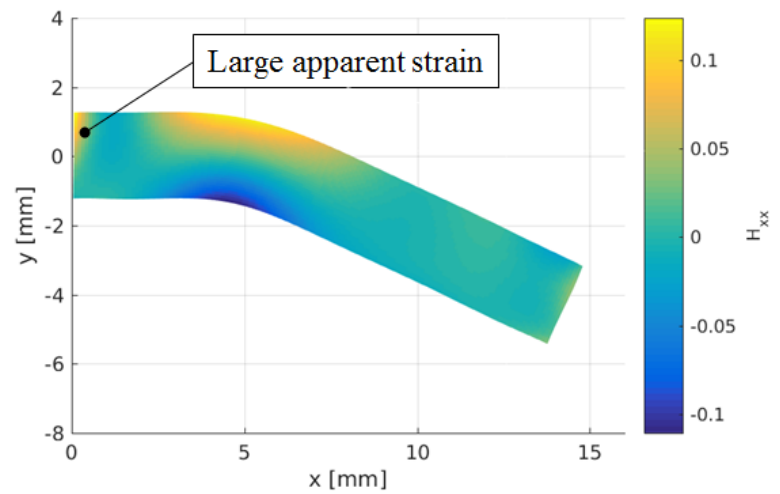

Fig. 2. Hencky strain $H_{x x}$, polynomial approximation.
The second evaluation variant shows the same example using B-spline approximation of the displacement data. The distribution of $H_{x x}$ at the specimen surface is given in Fig. 3. Good results at large and slightly deformed sections at the whole specimen surface are achieved. The reason is the better local adaption of the displacements by means of splines instead of global polynomial surfaces.

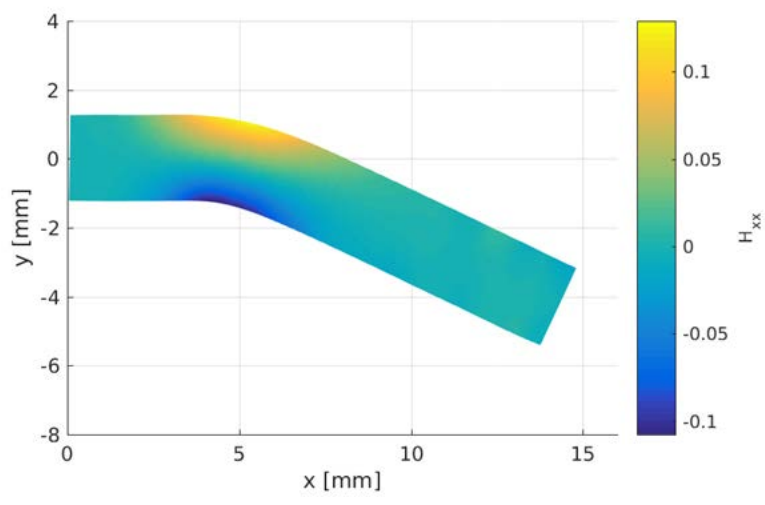

Fig. 3. Hencky strain $H_{x x}$, cubic B-spline approximation.

\section{Concluding remarks}

- Deformation field analyses using DIC with AZ31 specimens were demonstrated.

- Using B-spline approximation of the displacement data leads to better results regarding the determined strain distribution compared to the method using global polynomial surfaces.

- Different levels of deformation in tension and compression sections were determined.

\section{References}

[1] Dudamell, N.V., Ulacia, I., Gálvez, F., Yi, S., Bohlen, J., Letzig, D., Hurtado, I., PérezPrado, M.T., Influence of texture on the recrystallization mechanisms in an AZ31 Mg sheet alloy at dynamic rates. Mat. Sci. and Eng. A, 532, 2012. pp. 528-535.

[2] Knezevic, M., Levinson, A., Harris, R., Mishra, R.K., Doherty, R.D., Kalidindi, S.R., Deformation twinning in AZ31: Influence on strain hardening and texture evolution. Acta Materialia, 58, 2010. pp. 6230-6242.

[3] Kirbach, C., Lehmann, T, Stockmann, M., Ihlemann, J., Digital Image Correlation Used 

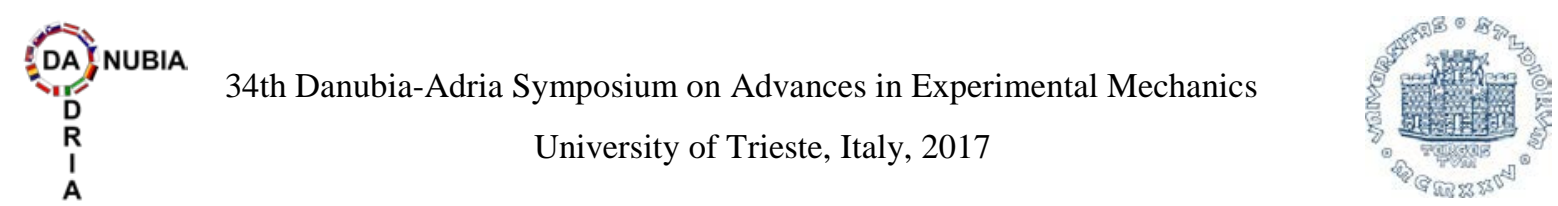

for Experimental Investigations of $\mathrm{Al} / \mathrm{Mg}$

Compounds. Strain, 51, 2015. pp. 223-234. 